\title{
The Hungarian pear germplasm as source of genetic variability for breeding programmes
}

\author{
Szabó, T. ${ }^{1}$, Labuschagne, I.F. ${ }^{2}$, Musacchi, S. ${ }^{3}$, Nyéki, J. ${ }^{4}$, Tornyai, J. ${ }^{4}$, Soltész, M. ${ }^{4}$ \& Szabó, Z. ${ }^{4}$ \\ ${ }^{1}$ Fruit Research and Extension Institute Úffehertó Hungary \\ ${ }^{2}$ Colors Fruit, P.O. Box 499 Paarl South Africa \\ ${ }^{3}$ Dipartimento Colture Arboree, University of Bologna, Bologna, Italy \\ ${ }^{4}$ Institute for Research and Development, University of Debrecen Hungary
}

\begin{abstract}
Summary: The Hungarian pear collection (Pyrus communis L.) consists of 423 genotypes distributed over seven genebanks in Hungary. This is one of the most extensive collections of native and cultivated pears found in Eastern Europe and includes a wide range of genotypes with small size fruit (referred to as "Miniature pears"). Based on the in situ and ex situ measures taken by governmental and other institutions for fruit tree conservation in Hungary, an overview is given on some activities regarding areas of Pyrus collection and genebanks where pears are collected and grown. Descriptions of traits of miniature pears found in Hungarian genebanks for the interest of genetic characterization and breeding are presented.
\end{abstract}

Key words: Gene conservation, natural genetic resources, landraces, genetic diversity

\section{Introduction}

Collection and establishment of basic material to be used for gene conservation activities ex situ and to serve as a starting point for the "modern" commercial fruit industry in Hungary started during the $18^{\text {th }}$ century. This process was maintained up to a certain point whereafter changes in land ownership (establishment of state farms and cooperatives), where collections were held after the world war, contributed to eradication of old orchards and increased the speed of exploitation of the natural genetic reserves of fruit species, including the European pear (Pyrus communis L.). The removal of standard trees on the common used pastures and woods of traditional communities was evident as well as the so called "wild orchards" and / or forests that was threatened with losses due to the extension of cultivated land.

It was anticipated that continued high-input and intensive agricultural practices would lead to further degradation of ecologically sensitive areas of which over 3 million hectares have been identified in Hungary (Holly et al., 2009). The need to collect the natural genetic resources became urgent around the middle of the 1970s. Identification and collection of historically and traditionally grown fruit, evolved from wild populations in the forests (landraces) were the first steps for the conservation and promotion of fruit species in situ. These landraces were adapted to local environmental and soil conditions and included interesting and valuable pears (179 genotypes).

The scope of many of these collections did not fulfill the role of traditional genebanks, because of an immediate focus on commercial production and marketing standards. Consequently, the fate of in situ collections was endangered and structured genebank collections became more important.
The need for additional conservation methodologies became apparent and traditional genebank activities have been complemented by two forms of conservation i.e., "On-farm" conservation within a given landscape and agricultural district where locally adapted genotypes were the most stable varieties, the result of long-term selection and adaptation processes (Holly, et al., 2009). Since 1996, this project formed part of the global programme co-ordinated by International Plant Genetic Resources Institute (IPGRI) that aimed at the establishment of a scientific base for on-farm conservation (Holly \& Szekely, 2001). The role of home gardens was also investigated in the maintenance of crop genetic diversity (Holly \& Szekely, 2001). The principal purpose of this programme was to conserve the original genetic composition and integrity of landraces, old cultivars and local varieties (Holly et al., 2009). This programme was based on the multiplication of locally adapted genotypes in selected districts where the climatic and edaphic conditions are similar to those of the places of origin. Research from Hungary highlights the case of home gardens as a source of crop genetic diversity in addition to their traditional role in families' food security (Eyzaguirre \& Bailey, 2007).

The conservation of natural genetic material with special or valuable horticultural characters for future plant breeding can be carried out in suitable genebanks. Genebanks normally contain obsolete varieties, local varieties, improved and cultivated varieties, different breeders' lines and hybrids as well as regional land races, natural varieties and accessions of wild growing trees. From 1990 onwards, efforts have been made to establish a national base collection and a central database for native genetic resources in Hungary (Holly \& Szekely, 2001). 


\section{Pear collection in Hungary}

\section{Regions}

The majority $(60 \%)$ of the pear accessions originated in the western part of Hungary (Transdanubia), because this region is more coincident with traditional pear cultivation. The largest collections of pear genotypes were found in seven Hungarian growing regions:

1. North-Transdanubian region: Counties include Fejér, Komárom, Veszprém, the northern part of the Györ-Sopron county and the districts of Budapest. A significant number of pear accessions were collected at the Danube river bend and the island of Szigetköz towards the plateau and in the vicinity of the Velence lake. A considerable part of the collection (about $15 \%$ ) was found in this region.

2. Western Transdanubian region: Vas, Zala counties and the western and south-western part of GyörSopron county. The majority of accessions have been collected around the towns of Kőszeg, Szentgotthárd, Nagykanizsa and Zalaszentgrót. Genotypes found here represent $35 \%$ of all collected pear accessions.

3. Southern Transdanubian region: Baranya, Somogy, Tolna counties including the towns Pécs, Szekszárd, Dunaföldvár, where about $10 \%$ of the accessions originate.

4. Danube-Tisza-közi plane region: The area between the two rivers, Bács-Kiskun, Csongrád and Pest counties at the left bank of Danube river and the right bank of the Tisza river. Cegléd, Nagykörös, Kecskemét, Jánoshalma, Csongrád towns. These pears represent $10 \%$ of the collection.

5. Tiszántúli region: Békés, Csongrád, Szolnok, Hajdú-Bihar counties, and plane parts of SzabolcsSzatmár and Borsod-Abaúj-Zemplén counties. Especially around the towns Szolnok and Csongrád. Szeged was the source of $10 \%$ of the genotypes collected in the collection.

6. Nyírség region: Counties of Szabolcs-Szatmár, Borsod-Abaúj-Zemplén and Hajdú-Bihar, which represent the sandy soils of the N-E Great Plane. The upper part of the Tisza and Szamos rivers represent the $5 \%$ of the collection.

7. Upland region: Hilly northern part of the counties BorsodAbaúj-Zemplén, Heves and the central and NE Hungarian mountains, north-eastern parts of the counties Nógrád and Pest counties, around towns: Gönc, Gyöngyös and Nagymaros. They contributed $15 \%$ of the assortment. Table 1 summarises the distribution of pear accessions in Hungary.
Table 1. Distribution of Pyrus accessions in the Hungarian genebanks according to their distribution categories (2009).

\begin{tabular}{|l|c|c|}
\hline Distribution categor & No. of accessions & Per cent (\%) \\
\hline Cultivated varieties & 12 & 3 \\
\hline Accessions from older collections & 191 & 45 \\
\hline Land races & 179 & 42 \\
\hline Wild grown genotypes & 16 & 4 \\
\hline Hybrids & 25 & 6 \\
\hline Total & 423 & 100 \\
\hline
\end{tabular}

\section{Újfehértó Genebank}

In Hungary, the Plant Genebank Council and the Ministry of Agriculture commissioned the Research Station of Újfehértó to establish and maintain vegetative field collections of apple (Malus domestica), pear (Pyrus communis), quince (Cydonia oblonga) and medlar (Mespilus germanica) for long-term germplasm conservation (Szabó \& Csiszár, 2002). Collecting of genetic resources for this genebanks started in 1978. The large-scale collecting work was finished in 1982, and by 1983 a five hectare genebank was established in Ujfeherto, with two trees per accession, on M4, wild pear and quince rootstock (Szabó \& Csiszár, 2002). The number of accessions maintained in the genebank of Újfehértó, comprises of 706 apples, 423 pears and 55 quince genotypes.

Data recording for each accession starts in the first year of fruit bearing. Knowledge of the date of flowering and ripening, fruit characteristics and growing habit of the tree allows the identification of varieties and possible duplicates and are important reference for breeders and collectors. Table 2 lists the characters recorded on small sized pears referred to as "miniature pears" (Szabó \& Csiszár, 2002).

In Hungary, one of the major pests of pears is pear psylla (Psylla pyri) for which chemical control is very difficult and expensive. After six years of observation (1996-2001) seven pear accessions in the genebank showed no pear psylla

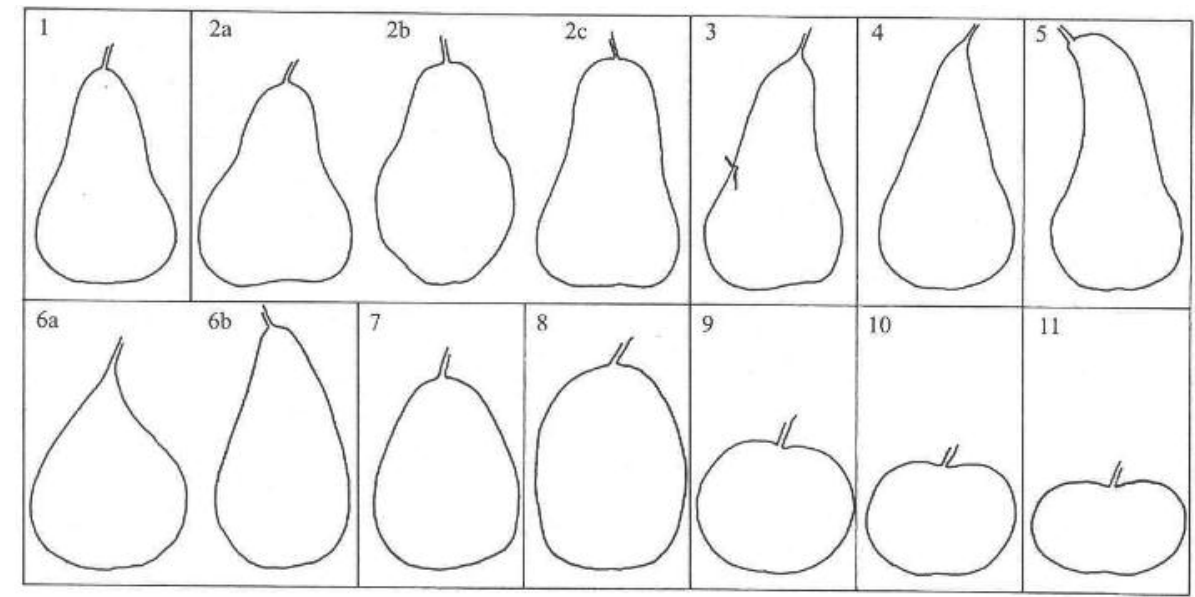

Figure 1. Fruit shapes of miniature pear collection in Hungary including 1. regular fruit shape; 2a-c. broadened shape; 3 . elongated; 4. very long; 5. bottle shaped; 6a-b. conic; 7. oval; 8. cylindrical; 9. globular; 10. flattened globe; 11 flat (Adapted from Göndör, 2000). 


\begin{tabular}{|c|c|c|c|c|c|c|c|c|c|c|c|c|c|c|c|c|c|c|c|c|c|c|c|c|c|c|c|}
\hline 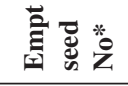 & 0 & $m$ & in & - & $N$ & $\sim$ & $m$ & $m$ & $\nabla$ & $m$ & $m$ & $\nabla$ & a & $N$ & 0 & $\nabla$ & $\nabla$ & $n$ & & $m$ & $a$ & $m$ & $n$ & $m$ & & $m 0$ & $\infty$ \\
\hline 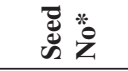 & & 으 & 으 & $\subseteq$ & & 으 & 인 & 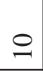 & 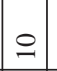 & 은 & $a$ & ㅇ & 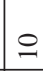 & 잉 & 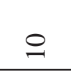 & 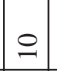 & $a$ & $\sigma$ & 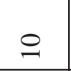 & $a$ & 의 & 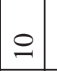 & 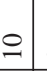 & $a$ & e & 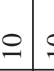 & 은 \\
\hline 总 & $\sum_{n}^{\circ}$ & 岕 & 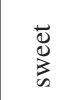 & 这 & & $\begin{array}{l}\stackrel{\square}{0} \\
\vdots \\
\vdots\end{array}$ & 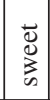 & $\begin{array}{l}\overrightarrow{8} \\
\bar{s} \\
\bar{s}\end{array}$ & \begin{tabular}{|l|} 
\\
0 \\
$\vdots$ \\
$\vdots$ \\
$\vdots$
\end{tabular} & 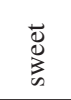 & $\begin{array}{l}\vec{y} \\
\bar{y} \\
\bar{s}\end{array}$ & 竞 & 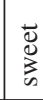 & 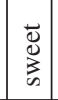 & 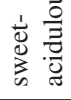 & \begin{tabular}{|l|} 
\\
\\
$\vdots$ \\
$\vdots$ \\
$\vdots$
\end{tabular} & 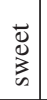 & ( & 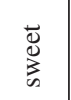 & $\begin{array}{l}\overrightarrow{0} \\
0 \\
\vdots \\
3\end{array}$ & $\overline{\mathbb{v}}$ & 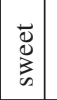 & ह & 3 & & & \\
\hline 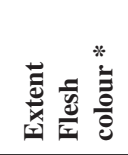 & 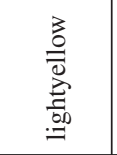 & 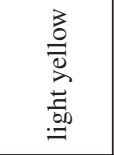 & 竘善 & 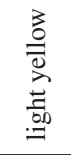 & 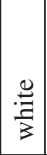 & 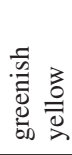 & 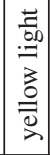 & 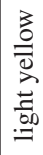 & 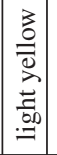 & 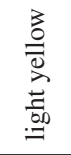 & 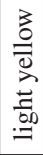 & 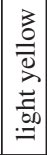 & 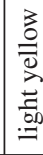 & 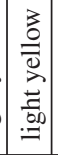 & $\begin{array}{l}\stackrel{z}{0} \\
\overline{0} \\
\stackrel{0}{ \pm} \\
\stackrel{0}{0.00} \\
:=\end{array}$ & 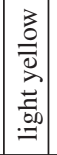 & 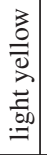 & 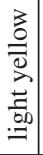 & $\begin{array}{l}3 \\
\stackrel{3}{0} \\
\overline{0} \\
\stackrel{0}{0} \\
\frac{0}{0.0} \\
=\end{array}$ & 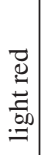 & 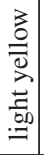 & 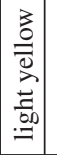 & 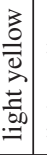 & 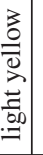 & & $\begin{array}{l}2 \\
0\end{array}$ & 离 \\
\hline 巳゙ & 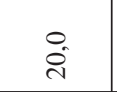 & $\tilde{n}$ & 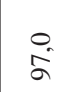 & in & & in & & $\begin{array}{l}0 \\
i \\
\end{array}$ & in & in & $\begin{array}{l}0 \\
\text { ì }\end{array}$ & $\mid \begin{array}{l}n \\
\stackrel{n}{2} \\
0\end{array}$ & & I & $\stackrel{\circ}{\varrho}$ & \begin{tabular}{|c}
0 \\
in
\end{tabular} & $\begin{array}{c}0 \\
\dot{m} \\
m\end{array}$ & & $\stackrel{0}{0}$ & $\begin{array}{l}0 \\
\dot{a}\end{array}$ & $\begin{array}{l}0 \\
i \\
i\end{array}$ & $\begin{array}{l}0 \\
i j \\
f\end{array}$ & $\begin{array}{l}0 \\
\dot{a} \\
\text { in }\end{array}$ & $\begin{array}{l}0 \\
\vec{n} \\
\end{array}$ & & \begin{tabular}{c|c}
$\dot{m}$ & \\
$m$ &
\end{tabular} & : \\
\hline 总 & 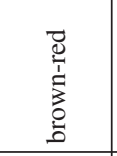 & 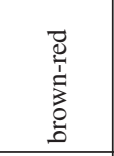 & 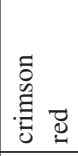 & 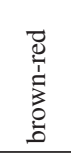 & 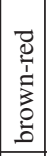 & 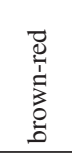 & 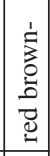 & 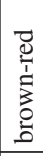 & 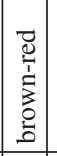 & $\begin{array}{l}\text { 品 } \\
\text { : } \\
\text { : } \\
\end{array}$ & 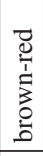 & \begin{tabular}{|l|} 
\\
8 \\
5 \\
5 \\
\end{tabular} & & & 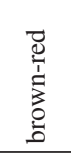 & 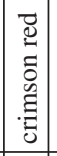 & 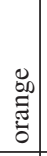 & & 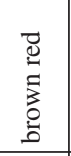 & 胥 & 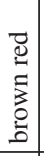 & 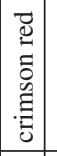 & 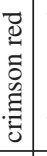 & 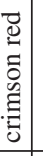 & & . & 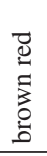 \\
\hline 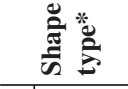 & $a$ & & 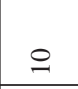 & $a$ & $a$ & $a$ & $a$ & $a$ & $a$ & $r$ & $a$ & 은 & $a$ & 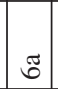 & $r$ & 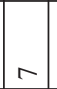 & $m$ & $a$ & $a$ & - & ते & 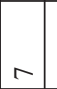 & $a$ & ฮ็ & - & $\overrightarrow{\mathrm{N}}$ & तิ \\
\hline $\begin{array}{l}\text { 音 } \\
\text { 产 } \\
\end{array}$ & $\stackrel{m}{-2}$ & â. & : & ठे & ठे & $\begin{array}{l}\infty \\
\stackrel{2}{0} \\
0\end{array}$ & $\begin{array}{l}2 \\
\hat{\sigma} \\
\end{array}$ & $\begin{array}{l}0 \\
\circ \\
0\end{array}$ & \begin{tabular}{|l|}
2 \\
0 \\
0
\end{tabular} & $\Xi$ & $\stackrel{8}{-}$ & $\begin{array}{l}8 \\
0 \\
\end{array}$ & oे & $\stackrel{g}{-}$ & $\hat{\sigma}$ & o. & $\stackrel{f}{-}$ & $\stackrel{\Omega}{.}$ & ठ̀ & $\begin{array}{l}\text { oे } \\
\text {. }\end{array}$ & \pm & $\begin{array}{l}2 \\
\text { oे }\end{array}$ & ڤ̊ & $\stackrel{2}{\Xi}$ & 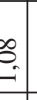 & $\exists:$ & $\Xi$ \\
\hline 㯊 & 8 & $\stackrel{\infty}{q}$ & fo & त̂ & § & $\begin{array}{l}\text { के } \\
\text { î }\end{array}$ & $\mid \begin{array}{c}1 \\
f \\
f\end{array}$ & $\begin{array}{l}0 \\
\dot{m} \\
\end{array}$ & $\ddot{n}^{2}$ & $\bar{n}$ & $\begin{array}{l}\infty \\
\vdots \\
m \\
m\end{array}$ & \begin{tabular}{|l}
$n$ \\
$n$ \\
$n$ \\
$n$
\end{tabular} & à & î & $\begin{array}{l}\infty \\
\dot{q} \\
\dot{q}\end{array}$ & 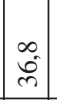 & $\begin{array}{l}0 \\
\dot{8}\end{array}$ & 文 & m & के & $\begin{array}{l}0 \\
\dot{a} \\
\dot{q}\end{array}$ & ले & 冓 & $\vec{y}$ & 6 & 资 & $\begin{array}{l}\text { î } \\
\text { in }\end{array}$ \\
\hline $\bar{E}$ & & $g$ & $\begin{array}{l}\infty \\
\substack{0 \\
\infty}\end{array}$ & $\vec{g}$ & & ले & $\begin{array}{l}0 \\
\dot{f} \\
\end{array}$ & $\begin{array}{l}0 \\
\dot{m} \\
\end{array}$ & \begin{tabular}{|c|} 
\\
aे \\
\end{tabular} & $\therefore$ & $\stackrel{m}{+}$ & $\begin{array}{c}0 \\
\dot{m} \\
m\end{array} \mid$ & \begin{tabular}{|l}
1 \\
$\dot{0}$ \\
$i$ \\
\end{tabular} & \begin{tabular}{|c|}
$n$ \\
$n$ \\
$m$ \\
$m$
\end{tabular} & $\begin{array}{c}m \\
\infty \\
m \\
m\end{array}$ & \begin{tabular}{|c|}
1 \\
$\tilde{n}$ \\
$\tilde{n}$
\end{tabular} & ?. & $\begin{array}{l}0 \\
\infty \\
\infty \\
\infty\end{array}$ & 宛 & $\begin{array}{l}\dot{+} \\
\dot{m} \\
\dot{m}\end{array}$ & $\overrightarrow{\hat{f}}$ & $\begin{array}{l}n \\
\infty \\
\infty \\
\infty\end{array}$ & $\begin{array}{l}\dot{s} \\
\dot{q}\end{array}$ & 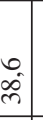 & 于 & $\vec{f}$ & 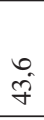 \\
\hline 䓂 & $\mathfrak{g}$ & if & & fं & & ले & 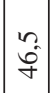 & m. & : & î́ & $\hat{m}$ & $\begin{array}{l}1 \\
\tilde{D} \\
\tilde{n}\end{array}$ & iे & $\mid \begin{array}{l}0 \\
\dot{m}\end{array}$ & $\dot{m}$ & $\overrightarrow{\tilde{m}}$ & $\mid \begin{array}{l}\vec{j} \\
\mathfrak{f} \\
\mathcal{f}\end{array}$ & $\begin{array}{l}n \\
? \\
q \\
q\end{array}$ & $\infty$ & $\begin{array}{c}\hat{\infty} \\
\stackrel{\rho}{\infty}\end{array}$ & $\hat{\jmath}$ & 尚 & 豖 & $\vec{q}$ & $\vec{y}$ & $q^{2}$ & 文 \\
\hline 惫 & in & $\stackrel{0}{\circ}$ & : & tóf & & $\hat{a}$ & :. & हे & $\hat{a}^{\prime}$ & ले & $\begin{array}{l}0 \\
\text { in } \\
\text { an }\end{array}$ & î & ले & f̀ & $\frac{0}{m}$ & 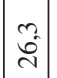 & $\mid \begin{array}{l}\infty \\
0 \\
0\end{array}$ & 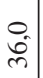 & 完 & $\begin{array}{l}\text {. } \\
\dot{m}\end{array}$ & $\frac{2}{7}$ & 하 & है & $\begin{array}{l}\text { in } \\
\text { in }\end{array}$ & 寸 & $\begin{array}{c}\hat{y} \\
\dot{y}\end{array}$ & $\begin{array}{l}\text { î } \\
\text { in }\end{array}$ \\
\hline 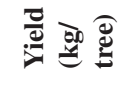 & $\infty$ & f & के & in & $\bar{m}$ & $\stackrel{\infty}{+}$ & $\vec{i}$ & $?$ & $\cong$ & $m$ & $\stackrel{\circ}{+}$ & $\begin{array}{l}\mathfrak{r} \\
\mathfrak{i}\end{array}$ & $\hat{i}$ & $\hat{i}$ & $\therefore$ & $I$ & $\stackrel{\sim}{\sim}$ & $\begin{array}{l}0 \\
v^{\circ}\end{array}$ & ते & $\stackrel{m}{m}$ & $\vec{m}$ & $\stackrel{ \pm}{0}$ & ì & $\vec{m}$ & $m$ & $\vec{i} \mid \hat{c}$ & $\dot{m}^{2}$ \\
\hline 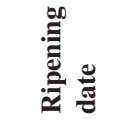 & 当 & $\frac{9}{3}$ & $\stackrel{\leftrightarrow}{=}$ & $\stackrel{\text { त̇ }}{\stackrel{\Xi}{>}}$ & & $\stackrel{\stackrel{\ominus}{g}}{\stackrel{B}{>}}$ & 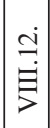 & $\stackrel{m}{\stackrel{5}{\Rightarrow}}$ & 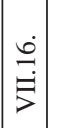 & ப્વ & 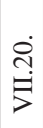 & 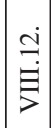 & 官 & 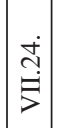 & $\stackrel{\stackrel{n}{\dot{m}}}{\vec{\xi}}$ & 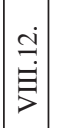 & $\stackrel{\dot{\vec{g}}}{\vec{\xi}}$ & $\dot{\dot{B}}$ & 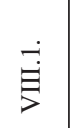 & $\stackrel{\overrightarrow{\vec{T}}}{\vec{\nabla}}$ & 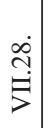 & 突 & $\begin{array}{l}\dot{0} \\
\dot{B} \\
\end{array}$ & 官 & & & 宊 \\
\hline 焉焉 & ? & $\mathcal{G}$ & 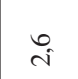 & i & 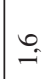 & i & $\stackrel{0}{-}$ & 3 & $\stackrel{\infty}{\infty}$ & $\stackrel{\partial}{=}$ & $\stackrel{\infty}{i}$ & $\stackrel{0}{-}$ & $\begin{array}{l}0 \\
i\end{array}$ & $\mid \stackrel{ \pm}{-}$ & $\vec{i}$ & $\stackrel{ \pm}{-}$ & $\stackrel{+}{i}$ & $\vec{i}$ & $\stackrel{ \pm}{-}$ & $\therefore$ & $\because$ & $n$ & $\stackrel{2}{\cong}$ & $\stackrel{\infty}{\infty}$ & + & $\begin{array}{lll}0 & = \\
- & \end{array}$ & $\stackrel{ \pm}{=}$ \\
\hline 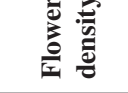 & 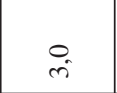 & i & $m$ & $\vec{m}$ & 4 & $m^{m}$ & $\begin{array}{l}0 \\
i\end{array}$ & $\stackrel{\circ}{\rightarrow}$ & $\vec{i} \mid$ & $\hat{i}$ & $m^{m}$ & $\vec{i}$ & $\stackrel{m}{m}$ & $\left|\begin{array}{c}\vec{i} \\
\mathrm{i}\end{array}\right|$ & लै & ì & लै| & ले & $\vec{i}$ & $\hat{i}$ & 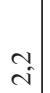 & $\stackrel{g}{\rightarrow}$ & ii & i. & $\hat{\mathrm{i}}$ & $\vec{i}$, & i \\
\hline $\bar{z}$ & 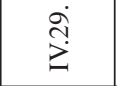 & $\stackrel{\infty}{\stackrel{i}{\geq}}$ & $\stackrel{\stackrel{N}{N}}{\geq}$ & $\stackrel{\vec{N}}{\grave{z}}$ & $\bar{z}$ & 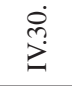 & 悹 & 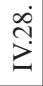 & $\mid \begin{array}{c}\hat{N} \\
\dot{z}\end{array}$ & ì̀ & $\dot{-}$ & 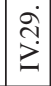 & ì & $\mid \begin{array}{c}\dot{0} \\
\stackrel{m}{2} \\
\dot{z}\end{array}$ & $\begin{array}{l}\stackrel{0}{1} \\
\stackrel{3}{Z}\end{array}$ & 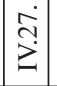 & ì่ & $\overrightarrow{>}$ & 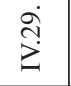 & $\dot{\vec{亠}}$ & 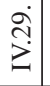 & 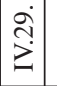 & $\begin{array}{c}0 \\
\stackrel{0}{0} \\
\grave{z}\end{array}$ & 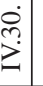 & 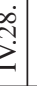 & 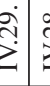 & 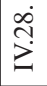 \\
\hline 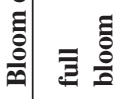 & \begin{tabular}{l}
$\dot{c}$ \\
\multirow{z}{z}{}
\end{tabular} & 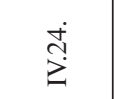 & 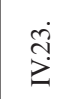 & $\begin{array}{l}\stackrel{d}{d} \\
\stackrel{Z}{Z}\end{array}$ & 6 & 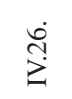 & ب⿱宀 & 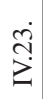 & $\mid \begin{array}{c}\text { ì } \\
\stackrel{z}{z}\end{array}$ & $\begin{array}{l}\stackrel{u}{c} \\
\stackrel{z}{z}\end{array}$ & $\overrightarrow{\hat{\lambda}}$ & 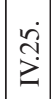 & $\begin{array}{l}\dot{n} \\
\stackrel{2}{z}\end{array}$ & $\mid \begin{array}{c}\hat{\imath} \\
\grave{Z}\end{array}$ & $\stackrel{\substack{\mathfrak{d} \\
己}}{\geq}$ & 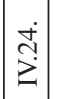 & 究 & 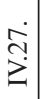 & $\stackrel{n}{a}$ & 完 & 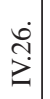 & 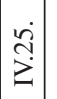 & 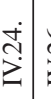 & 弪 & $>$ & 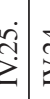 & $\stackrel{\stackrel{d}{d}}{\stackrel{2}{Z}}$ \\
\hline 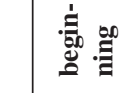 & 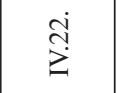 & กุ่ & $\frac{9}{z}$ & 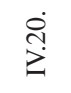 & 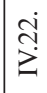 & הู่ & ָ̇i & $\stackrel{\infty}{\stackrel{\infty}{z}}$ & $\stackrel{\substack{\infty \\
\hdashline}}{\stackrel{2}{Z}}$ & $\dot{\Omega}$ & 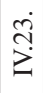 & $\stackrel{\dot{\vec{T}}}{\grave{z}}$ & 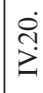 & $\begin{array}{c}\dot{a} \\
\grave{z}\end{array}$ & $\stackrel{9}{\grave{z}}$ & 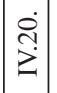 & 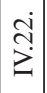 & 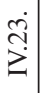 & $\stackrel{\dot{\vec{T}}}{\stackrel{\vec{P}}{-}}$ & $\stackrel{\substack{i \\
\geq}}{\geq}$ & 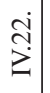 & $\mid \begin{array}{c}\dot{\vec{N}} \\
\dot{z}\end{array}$ & $\stackrel{2}{2}$ & $\begin{array}{c}\text { ì } \\
\text { ż }\end{array}$ & $\geq$ & $\overrightarrow{\mathrm{c}}$ & $\underset{\vec{N}}{\dot{\lambda}}$ \\
\hline 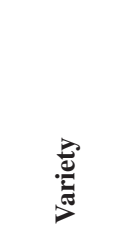 & 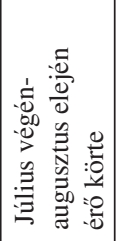 & 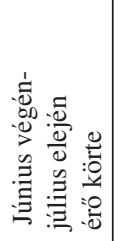 & 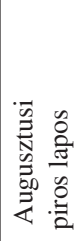 & 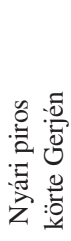 & 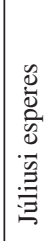 & 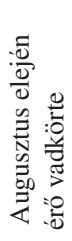 & 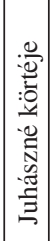 & 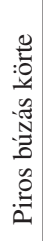 & 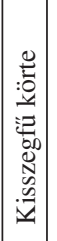 & 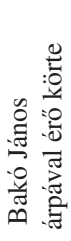 & 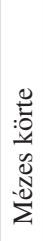 & 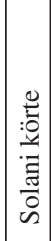 & 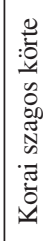 & 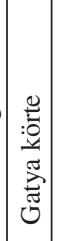 & 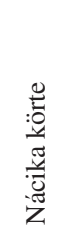 & 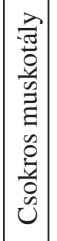 & 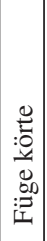 & 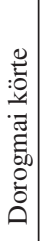 & 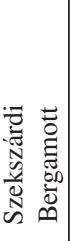 & 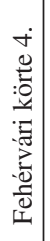 & 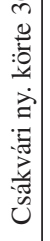 & 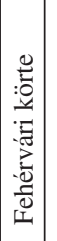 & 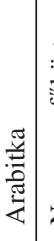 & Z & & 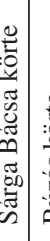 & 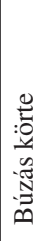 \\
\hline
\end{tabular}




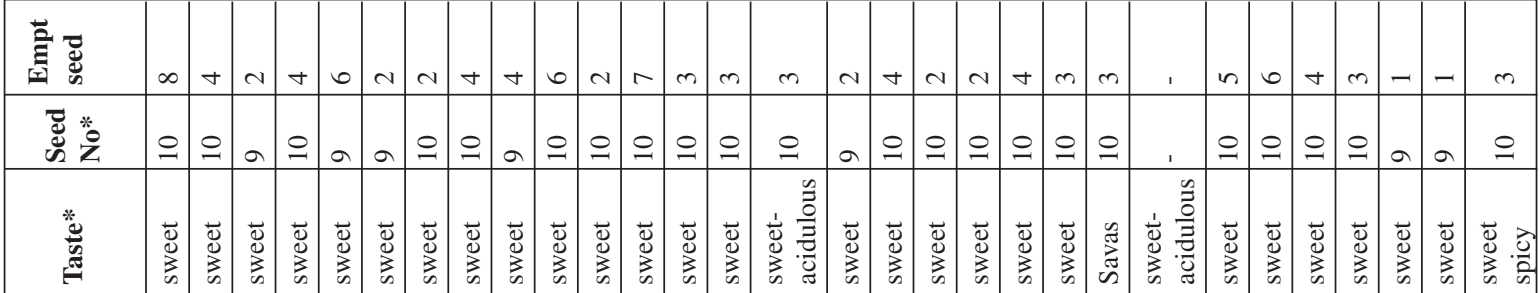

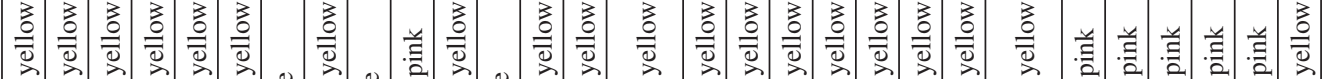

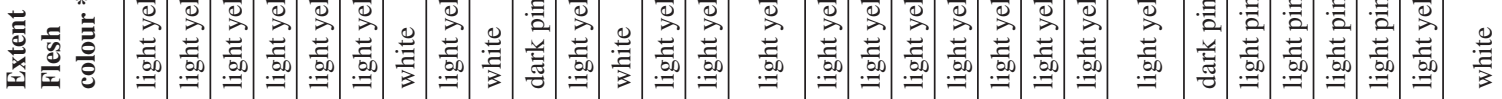

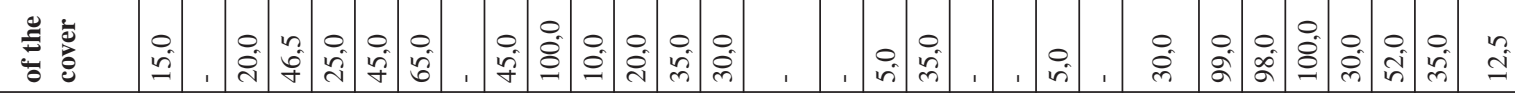

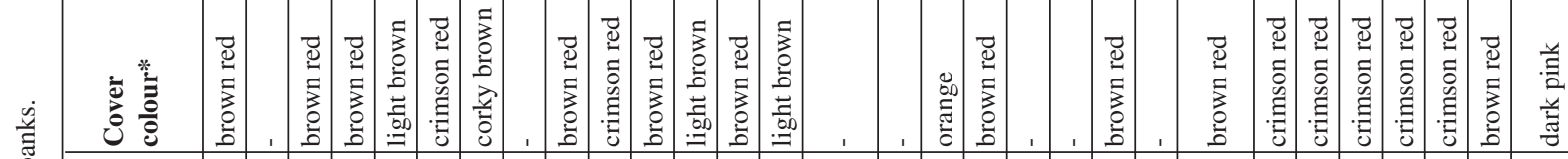

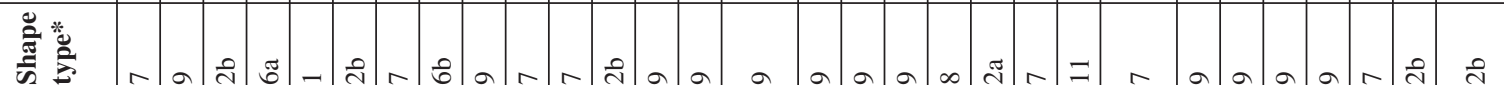

$\bar{\Xi}$

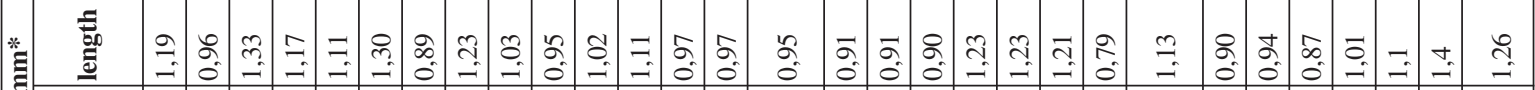

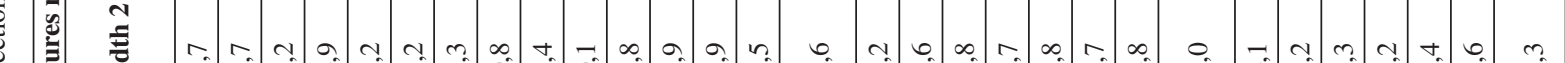

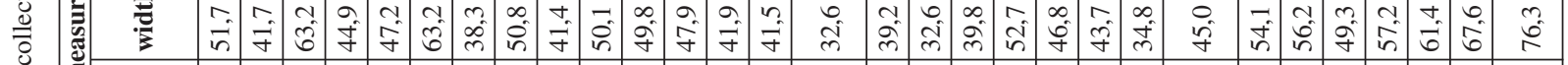

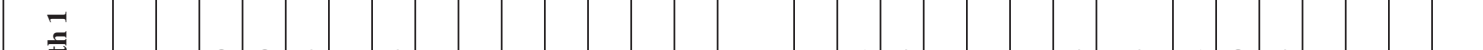

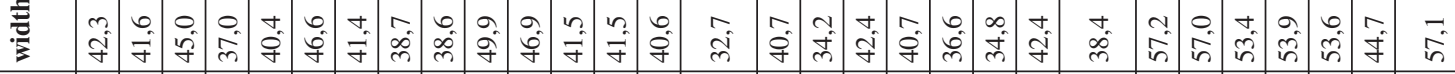

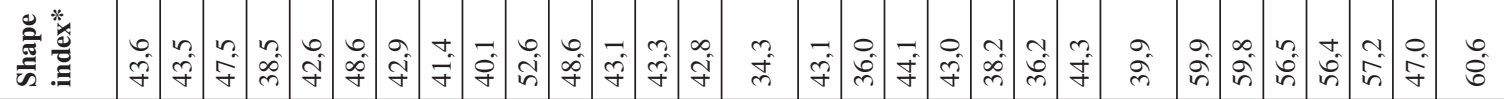

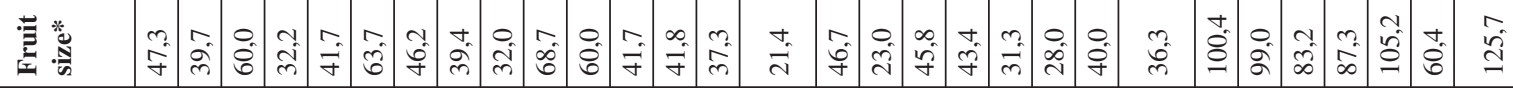

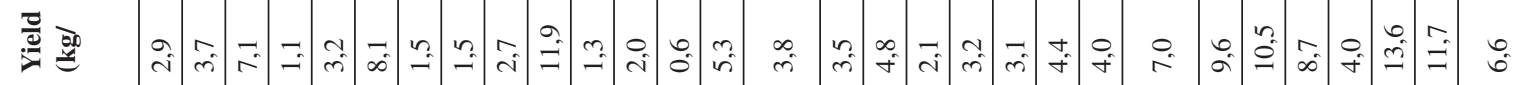

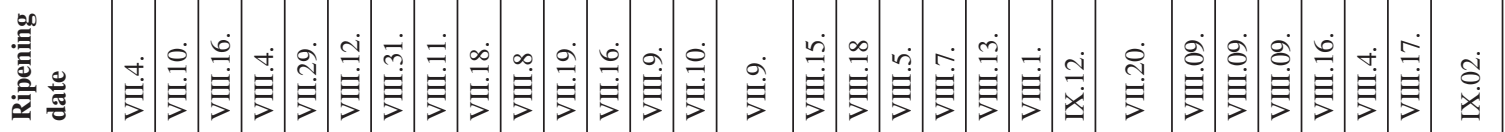

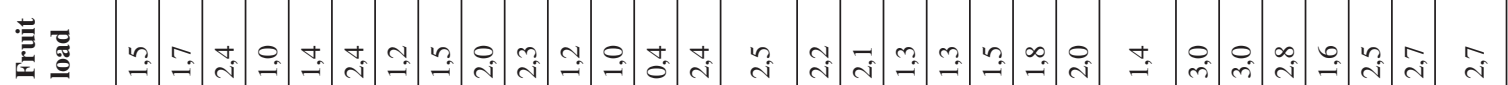

हैँ

它

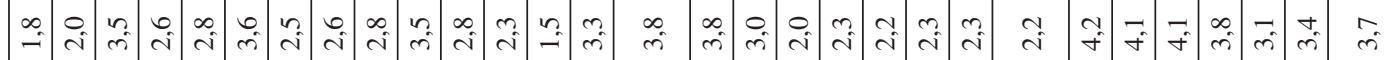
=

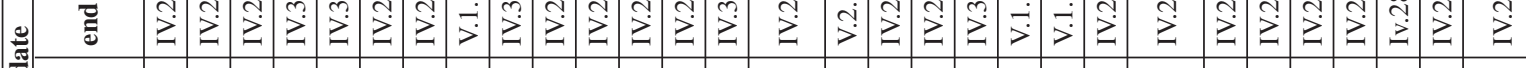

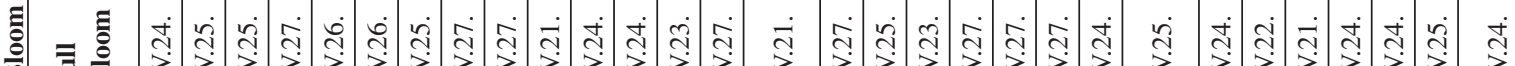

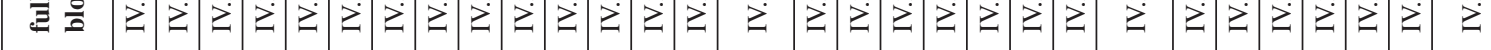

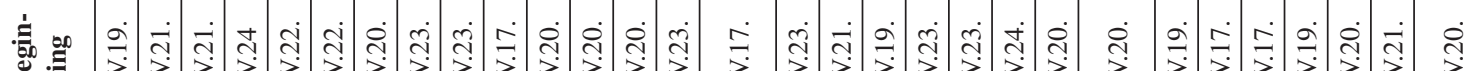

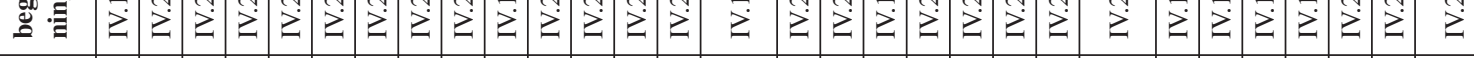

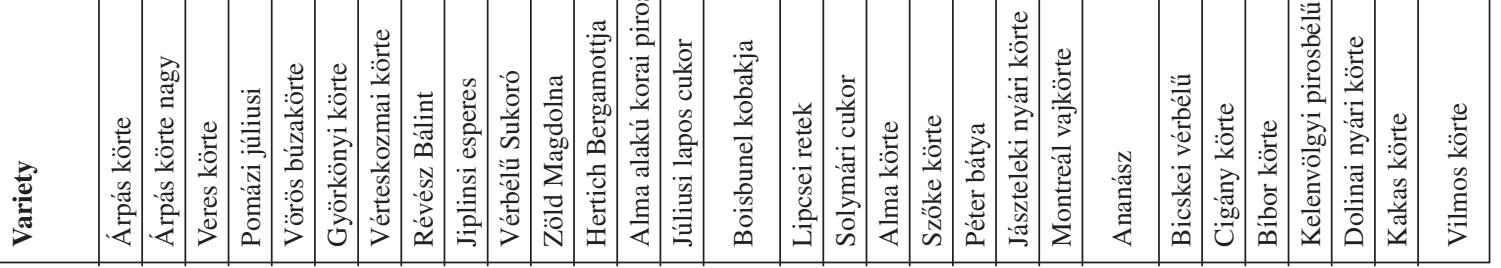




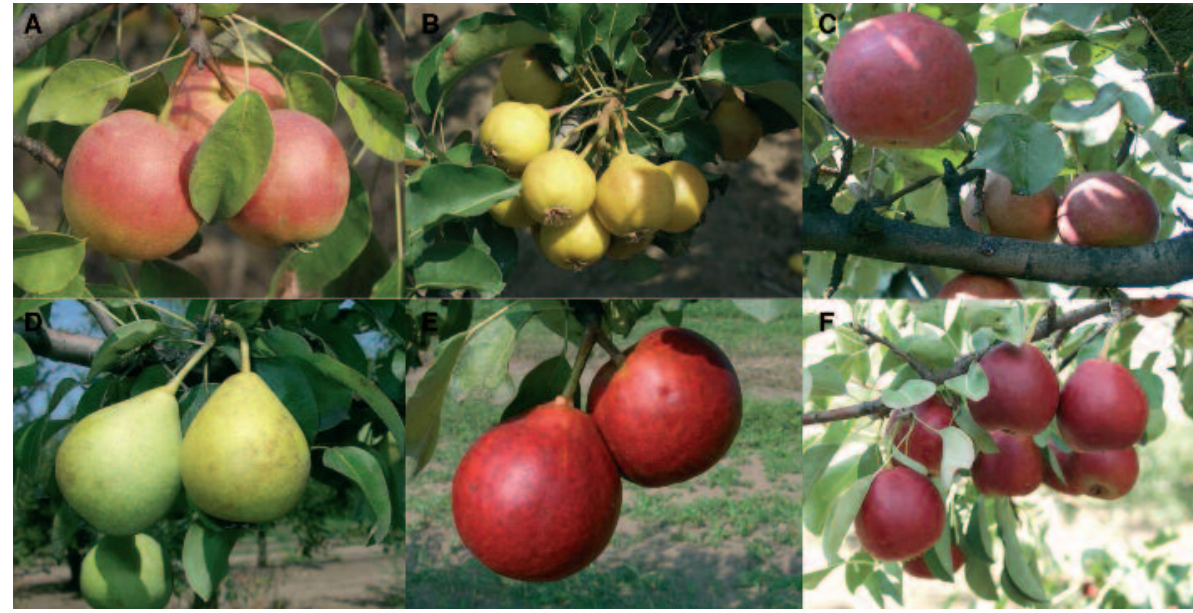

Figure 2. Miniature pear genotypes from Hungarian genebanks. A. Alma körte ("Apple pear"), B. Arabitka, C. Augusztusi piros lapos ("Flat, red August pear"), D. Bakó János árpával érő körte ("Ripening in the time of János Bakó barley”), E. Bíbor körte ("Purple pear"), F. Bicskei vérbélü ("Bloody core pear of Bicske")

damage (Bókoló körte (Dunaföldvár), Füge alakú körte (Velence), Nagyasszony körte Nyári Kálmán (Érd), Nyári körte (Dunaföldvár), Rozsnyári körte, Viki körte). Most of these varieties belong to the 'Sommer Christen Birne' type pears, except 'Füge alakú körte' and 'Viki körte' (Szabó \& Csiszár, 2002).

Most of the pear and quince varieties in this genebank are very susceptible to fire blight (Erwinia amylovora). In Hungary the infection risk is very high in the north eastern region because of the customary warm weather during blossom time (Szabó \& Csiszár 2002). This is the main reason why the Ministry of Agriculture decided to establish a second pear genebank at the Research Station of Fertöd in the northwestern part of Hungary (close to the Austrian border). All pear accessions from Újfehértó have already been propagated ( 2 trees/accession) in 2001, and they were re-planted in spring 2002. There are further plans to safetyduplicate the material and most of the pear varieties can already be found at the station of Keszthely, in western Hungary (Szabó \& Csiszár 2002). The two commercial pear cultivars that are the most important in Hungary is Bosc (32\%) and Williams (20\%). The other less important cultivars that are grown are Conference, Packham's Triumph, Clapp's Favourite and Hardenpont.

\section{Characterisation of miniature pear varieties from Hungarian genebanks}

In order to provide information to breeders and to identify specific donors to breeding programmes from the genebanks, evaluation and description of genotypes were performed and data collected for 57 miniature pears over a number of years (Table 2). In Hungary, flowering time for all miniature pears is around 17-24 April, full bloom between 21-27 April and end of bloom between 28 April and 1 May. Ripening date ranges between 4 July and 31 August, with some later genotypes of which two ripen in September and one in
October. Fruit size for the miniature pears is normal distributed, ranging from $9 \mathrm{~g}$ to $125 \mathrm{~g}$, with about half of the collection between $30 \mathrm{~g}$ and $50 \mathrm{~g}$, averaging $45.5 \mathrm{~g}$. The average length of the pears is $46 \mathrm{~mm}$ and ranges from 25 to $67 \mathrm{~mm}$. Accessions differ largely in flower density and fruit load, i.e., some genotypes show very low fruit load $(0.3 \mathrm{~kg} /$ tree $)$ and others up to $13.6 \mathrm{~kg} /$ tree on average for three years. Twelve accessions show $50 \%$ or more seed in fruit to be flat, empty and undeveloped.

From this collection, three pears have dark red internal colouring, four show light red flesh and five have white flesh. Other accessions all show light yellow flesh colour. Taste is sweet for most accessions, three have a more balanced sweet acid taste and one is acidic. External colour includes browns and reds that varies from brown red, crimson red to light red, orange red and pink. Colour coverage ranges from no coverage (17.5\% of genotypes) to near full or full coverage $(8.8 \%)$ and about half of the collection show between $10-40 \%$ of the fruit coloured. Fruit shape varies from regular to elongated, globular and flat, but most are globular (42\%), oval $(22 \%)$ or oblong ovate pyriform (14\%). Nine accessions are slightly elongated and only Szöke körte ("Fair pear") has flattened fruits.

\section{Short Descriptions of specific genotypes}

1. Alma körte (Apple pear): The variety has been collected on the southern slopes of the Mecsek mountain, around the commune of Boda in home gardens by the associates of the Horticultural Research Institute. The ripening time is the first week of August cc. three weeks earlier than Williams. The fruit are suitable for fresh consumption, but have short shelf life, lasting a few days before getting soft. Fruits are small (40-50 g), $41 \mathrm{~mm}$ long, $45 \mathrm{~mm}$ wide, like a fattened globe (shape ratio: 0.9). The peduncle is long and thick, reddish, thickening near the fruit base. Fruit skin is smooth, medium thick, greenish yellow with crimson area covering $30-50 \%$ of the fruit. Clear, brown lenticels are abundant. The flesh is whiteyellow, semi-hard, juicy, but mealy at full maturity. Around the follicle stony granules occur. It tastes sweet without a particular flavour. Inside, in the closed cavity many full seeds are set. Trees are vigorous, initially upwards growing, slender, developing towards a globular canopy. The branching system is favourable, many short fruiting shoots are developed. Shoots are light brown, thin, scarcely spotted with lenticels. Buds are closely fitted to the 
shoots, bud scales are clear and downy. Blooming ensues somewhat earlier than Williams, with mediocre intensity.

2. Arabitka: Late June and early July is the time of maturity for fresh consumption of this summer pear. Fruit is small $(30-40 \mathrm{~g}), 39 \mathrm{~mm}$ long and $40 \mathrm{~mm}$ wide. The flattened globe and at the peduncle, the fruit is bulging (shape index 0.9). The peduncle is long and fleshy, light brown to reddish brown on greenish yellow background. Lenticels are light and scattered. Fruits appear in bunches on the tree. The skin is smooth with a lemon green ground colour, occasionally with some reddish brown spots on the sunny side. Brown lenticels are scattered on the surface. The flesh is white or yellowish, juicy and melting. The taste is sweet and the aroma is weak. Picked before full maturity, the fruits are transported and kept for some days on the shelf. The follicles are closed and enclose many seeds. Trees are vigorous, the initial pyramid shape turns into a more spreading growth habit. The branching system and the distribution of fruiting structures are good. The shoots are thick and stout, brownish, scattered with light lenticels. The buds are of mediocre size, closely adherent to the shoot. Early blooming, 1-2 days earlier than Williams. Flower density is very good and fruit set is excellent.

3. Augusztusi piros lapos (Flat, red of August): Ripening in early August, suitable for fresh consumption and becomes mealy a few days after picking, therefore it cannot be stored for long periods. Fruit is medium size (around $70 \mathrm{~g}$ ), $48 \mathrm{~mm}$ long, 53 $\mathrm{mm}$ wide, shape index: 0.9. The peduncle is long, green, and thick, even more at the fruit base. The fruit skin is slightly undulate, occasionally smooth, medium thick, its colour is light green covered almost entirely by a purple tinge with a slight waxy layer. Lenticels are of medium density, greenish yellow or purple. The fruit flesh is dark-pink or purple, in some years light red, its consistency is semi-firm, juicy, sweet, with weak aroma and less conspicuous stony granules around the follicles. The inner cavity is closed and contains many seeds. Trees are vigorous, crown is medium dense, canopy is oblong. The shoots are medium long, medium thick, light reddish brown. Small clear lenticels are abundant. Buds are small, closely fitting to the shoot or slightly detached. Early blooming a few days before Williams with mediocre flower density. This pear might be susceptible to pear leaf hopper.

4. Bakó János árpával érö körte (Ripening with barley of János Bakó): Ripening at end of July to early August. For fresh consumption, however, the fruit gets mealy soon at full maturity. Fruit size is small (30-50 g), $47 \mathrm{~mm}$ long, $40 \mathrm{~mm}$ wide, shape index: 1.2 (oval). Appearance is attractive with a smooth finish. The rind is medium thick, lemon yellow and red colour may cover the sunny side up to one quarter of the surface. The skin is dry to the touch, smooth with many clear lenticels. Peduncle is long, thin, reddish or yellow, with no depression at the fruit base. The flesh is medium firm, a little juicy, pale yellow, sweet, medium scented. Some stony granules occur. The follicles are partly closed, star-shaped with many seeds. Trees are medium vigorous, the canopy is medium dense with a globular crown. Shoots are medium long and medium thick with dark reddish-brown colour and spare lenticels. Buds are medium large, loosely fitting to the shoot. Blooming date is one or two days after Williams. Flower density is intermediate.

5. Bíbor körte (purple pear): For fresh consumption, fruits are picked in early to mid August. Fruit size is intermediate (70-100 g), $50 \mathrm{~mm}$ long, $57 \mathrm{~mm}$ wide, flattened and in some years globular. The shape index is 0.9 . The surface is slightly undulating, the skin is thick. The ground colour is lemon yellow, but the purple cover colour may dominate. The surface is densely scattered with brown lenticels especially on the calyx end. The peduncle is long and thick, green with lenticels and corky on the top. The semi-firm fruit flesh is juicy, mealy at full maturity, with stony granules around the seeds. A sweet taste is combined with a weak flavour. The follicles are closed and contain many seeds in the cavity inside. Trees are medium vigorous, the crowns are globular. The branching system is favourable, many short fruiting bodies are grown. Shoots are medium long. Early blooming, three days before Williams. Flower density and fruit load tend to be good.

6. Bicskei vérbélü (Bloody core of Bicske): Ripe at the end of July and early August for fresh consumption. Fruit size is intermediate ( $90-110 \mathrm{~g}$ ), $54 \mathrm{~mm}$ long, 60 $\mathrm{mm}$ wide, the shape index is 1.1 . The appearance is attractive, smooth and waxy. The peel is medium thick. The ground colour is light green covered almost entirely by the purple-red. Small, light lenticels are scattered on the surface. The fruit flesh is medium firm, melting juicy with some stone cells. Its colour is purple-red or dark pink. The sweet taste is combined with almost no aroma. The closed follicles contain many seeds. Trees are medium or highly vigorous with medium dense crowns of an elongated globular canopy. Shoots are medium thick, light brown with few lenticels. Buds are intermediate and are loosely attached to the shoot. The flowering time is three days before Williams. Flower density and fruit set are excellent.

\section{Discussion}

From the total area of Hungary $\left(93,000 \mathrm{~km}^{2}\right)$ cca., only 4,500 hectares is suitable for pear production, because most of the cultivated pear cultivars originated from the cooler Western-European countries, thus requiring higher air 
humidity. Successful pear growing is carried out only in two regions of the country, i.e., in Zala county and in the Bodrogköz region. Presently the total pear orchard area in Hungary under production is 3,200 hectares and the average yearly crop is $25-30,000$ tons of which half of the total production is exported. To have a wider selection of pear cultivars that can be grown commercially in Hungary and to expand pear production in Hungary, the use of locally adapted genotypes in combination with "foreign" cultivars will play a major role in a directed breeding and selection process. It will also be important to introduce cultivars resistant to Erwinia amylovora if commercial pear production in Hungary.

World-wide, fruit depositories, genebanks and diverse fruit collections of historic, cultivated and wild fruit species in private, governmental and / or federal institutions, receive attention from organizations that play a role in conservation of genetic resources, but also from private collectors and private breeders.

Characterization of collections and accessions in genebanks help to choose important genotypes and set up a core collection to integrate characters in the breeding process. To secure the long term and efficient utilization of fruit genetic resources in Hungary, to ensure the availability of germplasm for research, and in order to secure a rich genetic base for breeders to develop new innovative fruit cultivars for the future, conservation of natural gene reserves and the protection of old fruit genotypes in genebanks is critical. To meet ever evolving consumer demands, unique qualities of obsolete varieties, land races and natural varieties will become more important in future breeding programmes. Presently no pear breeding is performed in Hungary.

\section{References}

Eyzaguirre, P. \& Bailey, A. (2007): International case studies and tropical home gardens projects: Offering lessons for a new research agenda in Europe. In: A. Bailey, P. Eyzaguirre and L. Maggioni (eds.) The Proceedings of a ECP/GR Workshop: Crop genetic resources in EU home gardens, 3-4 October, Ljubljana, Slovenia

Göndör Jné. (2000): A körte fajtahasználata és nemesítése. 102-150. In: Göndör Jné (ed.) Körte. Mezőgazda Kiadó, Budapest

Holly, L \& Székely, B. (2001): Assessment of crop diversity in Hungary: Possible indicators for genetic variation. Paper presented to the: OECD Expert Meeting on Agri-Biodiversity Indicators, 5-8 November 2001 Zürich, Switzerland

Holly, L., A. Simon, I. Már, G.M. Csizmadia, Z. Kollár \& Hock, Z. (2009): Inventorying and on-farm Maintenance of Hungarian Landraces. In: M. Veteläinen, V. Negri and N. Maxted (eds.). Bioversity Technical Bulletin No 15. European landraces: On farm conservation management and use

Szabó, T. \& Csiszár, L. (2002): Research and Extension Centre for Fruit Growing, Újfehértó, Hungary. In: L. Maggioni, M. Fischer, M. Lateur, E.-J. Lamont and E. Lipman (eds.) Report on a ECP/GR Working group on Malus / Pyrus Second Meeting 2-4 May, Dresden-Pillnitz, Germany 